\title{
EFFECT OF BIOSTIMULANTS REMIDIATION SUBSTANCES (BRS) ON SEED GERMINATION AND SEEDLING GROWTH OF SOME SUGAR BEET CULTIVARS UNDER STRESS CONDITIONS
}

Helaly, M.N.*; M.T. Saker*; Z.E. Mohamed*; A.E. Allam ${ }^{\star *}$ and M. A.Ghaly**

* Dept. of Agric. Botany, Fac. of Agric., Mansoura Univ. Egypt.

** Sugar Crops Research Inst., Agric, Res. Center (ARC), Giza, Egypt.

\begin{abstract}
Two newly cultivars from sugar beet BBeta vulgaris,L; Chenopodiaceae Pleno(C1) and Plever(C2)\} were used to study the effects of certain antioxidant application on elevating the harmful effects of drought stress condition on seed germination as well as seedling growth and its constituents Mannitol levels and/or Ascorbic;AsA , Salslic; SA, Humic; HA and seaweed; SWE were examined as biostimulants remediation substances (BRS).

Analysis of variance showed a significant effects of Mannitol level and/or the BRS used $(P<0.001)$ on the rate of germination as well as fresh and dry weights of the seedling. The reduction in germination \% and the increase in time required for seeds to germinate due to stress was observed in both sugar beet $\mathrm{cv}(\mathrm{s})$ studied. However, $\mathrm{cv}(2)$ was more susibtable than $\mathrm{cv}(1)$ in this respect.

Germination was progressively inhibited by an increase in mannitol level in both $\operatorname{cv}(\mathrm{s})$. The strongest inhibition occurred at the third mannitol level $(0.3 \mathrm{M})$ in $\operatorname{cv}(2)$. However, cv(1) did not exert any significant effect on ultimate germination \% under the $2^{\text {nd }}$ mannitol level. Increasing mannitol level was associated with a marked reduction in AsA, glutathione; GSH, catalase;CAT, guaiacol peroxidase;GPOD and superoxide dismutase;SOD as well as total carbohydrates and total $\mathrm{N}$ concentrations, whereas, increased that of $\mathrm{H}_{2} \mathrm{O}_{2}$ and proline as well as T.S.S. and osmotic pressure of the leaves and roots in both seedling $\mathrm{cv}(\mathrm{s})$.

BRS decreased concentrations of $\mathrm{H}_{2} \mathrm{O}_{2}$ and proline whereas, increased that of AsA and GSH as well as CAT, GPOD and SOD in the development seedling of both cV (s). Treatment with SWE showed an additive effects to that of stress treatments on increasing non-enzymatic and enzymatic antioxidants concentrations in both $\mathrm{CV}$ (s). Cv (1) showed, in general higher concentrations of AsA and GSH as well as CAT, GPOD and SOD than that of $\mathrm{cv}(2)$.

The interactions treatments showed that any of the BRS used elevating the harmful effect drought stress caused by increasing mannitol level up to 3 rd one (3.0 $M)$. Again the SWE followed with HA treatments were the best in this respect.

Keywords: Germination, BRS, stress, mannitol, SWE, AsA, Ha, SA, GSH, CAT, GPOD, SOD,T.S.S, sugar beet.

\section{INTRODUCTION}

Water deficient is one of the main limiting factors of sugar beet production in arid and semi arid regions. It have serious impacts as germination and normal development of roots and shoot extension during germination. Moreover, it delaying seedling emergence prolonging critical growth period, increasing changes of seedling damage by pathogenitical and environmental factors. Seed germination and early seedling growth are the
\end{abstract}


stages most sensitive in sugar beet and it is related with genetical and environmental factors as well as seed pretreatments effects. It is most important for determining seed quality.

On the other hand, storage reserves within the seed are slowly depleted causing a decrease in seedling survival and growth vigour. Studies on abiotic stress tolerance in sugar beet have been undertaken for the identification of physiological and environmental factors to decide the ultimate crop yield (Tugnoli and Bettini, 2001).

It was suggested that, nutrient solution containing osmotic agent such as mannitol, and polyethylene glucose, PEG could be used in screening for drought tolerance in growing seedlings (Ghoulam and Fares, 2001). Osmotic adjustment in tolerant plant helps maintain leaf metabolism and root growth at relatively low leaf water potential by controlling turgor pressure in the cells.

Improve stress capacities of some existing varieties using biochemical's was reported by many investigators (Clapp, et al., 2002; Panda and Khan, 2003; Zhao and Qin, 2004 ; Tang and Newton, 2005 ; Nabati, et al., 2005 ; Faust, 2006 and Ozdoba, 2006).

The effects of BRS on controlling free radicals levels are considered to be the way of plant to tolerate stress ( Chattopadhyay et al., 2002) . Polyamines (PAs) greatly polentiate the effects of stress by enhancing reactive oxygen species; ROS generation during photosynthesis (Borsani et al., 2001) used as markers of physiological stress (Tang and Newton , 2005) and play a role in antioxidative system and protect membrance from peroxidation in in vitro cultures and induced adaptation to stress ( Mishra et al., 2003)

The effects of two newly sugar beet cv (s) seed pretreatments with some BRS on the germination and seedling growth under stress agent (mannitol levels) was studied with an aim to choose an evaluation procedure for the identification of sugar beet tolerant to water stress at germination and early growth stages.

\section{MATERIALS AND METHODS}

Germination experiments were carried out at the laboratory of the sugar crops research Institute, Agric, Res. Center (ARC), Egypt. During the seasons of 2007/2008 and 2008/2009 in an incubated condition at $25-27{ }^{\circ} \mathrm{C}$ for $16 / 18$ hours day/night condition using white fluorescent tubes .

Seeds of multigerm sugar beet (Beta vulgaris,L; Chenopodiaceae cvs; Pleno and Plever) were obtained from Sugar Crop Institute, Agric. Res. Center (ARC), Ministry of Agric., Egypt.

Seeds (fruits) were graded, standardized, washed, sterilized and dried at room temperature prior to the experiments of germination tests. Seeds were soaked for six $\mathrm{h}$ in the specific antioxidants used. Germination was took place in boxes $(37 \times 55 \times 13 \mathrm{~cm})$ at the rate of 100 seeds/box which containing perlite. Stress treatments were performed at 0.0 (distilled water ; control), 0.2 and $0.3 \mathrm{Ml}^{-1}$ concentrations of mannitol approximately corresponding to $1,-5$ and -7 bar osmotic pressure. The effects of certain antioxidants were 
examined in the presence or absence of mannitol : Ascorbic , Salslic, Humic and SWE were obtained from Sigma Comp. and used at the level of 250 $\mathrm{ppm}, 250 \mathrm{ppm}, 1 \mathrm{~m} / \mathrm{L}$ and $1 \mathrm{~m} / \mathrm{L}$ respectively. Boxes were watered with $1 / 2$ diluted Hoagland solution (Hoagland, and Arnon, 1950) for each different treatment.

The amount of these solutions was adjusted daily to keep a 2- $\mathrm{cm}$ level at the bottom . A factorial design with six replications was used for each treatment.

Germination \% was recorded after 21 days . The relative germination was determined daily by the following calculation: No. of germinated seeds in the stress medium/ No, of germinated seeds in control medium $\times 100$ (Smith and Dobrenz, 1987).

After germination, seedling were allowed to grow for 35 days from sowing. At the end of experiments, seedling characteristics including cotyledon fresh weight and its dry weight at $70^{\circ} \mathrm{C}$, root fresh and dry weight as well as root length were recorded.

Similarly, fresh and dry weights of roots and shoots (cotyledon+leaves) were calculated (Sadeghian and Yavari, 2004). Total carbohydrate content was estimated by the official methods (A.O.A.C. ,2000). Total nitrogen concentration (Nour, 1971), total soluble solide and osmotic pressure (O.P.) of the leaf sap (Slatyer and Mcllory, 1961) were estimated. Moreover, $\mathrm{H}_{2} \mathrm{O}_{2}$ (Velikova et al., 2000), ascorbic acid (Cakmak and Marschner , 1992), glutathione (El-Hoseiny Hanan,2008), and proline (Bates et al., 1973), also the activity of antioxidant enzymes; catalase (EC 1.11.1.6) according to Velikova et al., 2000, guaiacol peroxidase (EC.1.11.1.7) (Urbaremek et al., 1991) and superoxide dismutase EC 1.15.1.1. (Van Rossum et al., 1997) were determined.

\section{RESULTS AND DISCUSSION}

The data representing the germination \% and seedling characters of the two sugar beet $\operatorname{cv}(\mathrm{s})$ as affected by certain biostimulants remediation substances under drought stress condition using mannitol levels are recorded in Tables 3 and 4.

Decreased germination may be measured as delaying of emergence, reduction of the ultimate germination \% and /or both. Analysis of variance showed a significant effects of mannitol level and/or the BRS used $(P<0.001)$ on the rate of germination. The reduction in germination \% and the increase in time required for seeds to germinate due to water stress was observed in both sugar beet $\operatorname{cv}(\mathrm{s})$ studied. However, $\operatorname{cv}(2)$ was more susibtable than $\operatorname{cv}(1)$ in this respect. In addition, germination was progressively inhibited by an increase in mannitol level in both $\mathrm{cv}(\mathrm{s})$. The strongest inhibition occurred at the second mannitol level (0.3 M) in $\operatorname{cv}(2)$. However, $\operatorname{cv}(1)$ did not exert any significant effect on ultimate germination \% under the 2 nd mannitol level. The 3 rd level of mannitol significantly inhibited germination records of both $\mathrm{cv}(\mathrm{s})$.

The harmful effects of drought stress on germination may be due, mainly. to either the inhibition of colloidal inhibition of water occurred by seeds and/or unbalanced of osmotic water uptake occurred by germinated 
seeds (Helaly, 1972). Moreover, the effects of drought on altering the hormonal balances and decreasing endogenous cytokinins biosynthesis and auxin production (Schmidt,2005), decreasing water content, some nutrients uptake and root pull strength (Demir et al., 2004), decreasing antioxidants , a- tocopherol and carotenoids which necessary for PSIl (Hatung, 2004), increasing of lipid peroxidation and inactivation of enzymes (Smirnoff, 1995), disturbances in cell membrane components (Salisbury and Ross, 1992) were detected. Increasing free-radical groups activity which are major elements for chlorophyll degradations (Fletcher et al., 1988) and decreased the accumulation of reducing sugars within the plant tissues which decreased wilting resistance were also reported. Moreover, it was found that water stress did not induce an increase of ascorbic acid which not only quenches reactive oxygen but also, regenerates $\alpha$ - tocopherol (Schmidt,2005).

On the other hand, germinating the pretreated seeds may overcome all or part of the drought stress influence through the uptake of some of the surrounding solutes. Such seeds make the osmotic adjustment at the expense of time and risk of one or more from the physiological behavior reported above. Thus, the period elapsed for seed to germinate under stress condition was longer than that for seed germinated under normal condition (Table 3) . It is well known that, of the germinating seeds did not absorb enough water and solutes, they could not adjust to imposed stress influence.

Biostimulant Remediation Substances; BRS used showed a contributing influences on germination $\%$ and delaying their radical emergence (Tables 3 and 4). Seeds of the two cv(s) were responded to these substances differently depending on the level of mannitol, $\mathrm{cv}$ and the BRS used. Pretreated seeds with any of the BRS used, germination was took place over that of the non- pretreated seeds. The almost effective treatment on increasing germination\% was found with SWE in both $\mathrm{cv}(\mathrm{s})$ with the superiority of $\mathrm{cv}(1)$; more resistance than $\mathrm{cv}(2)$. It is interesting to note that, the pretreatment with either of BRS used alleviated the harmfull effects of the high mannitol level on germination of cv (1) as well as cv (2). In addition, pretreated seed with SWE and Humic acids; HA germinated faster than did those pretreated with Ascorbic, Salcilic and distilled water in a descending order. Final germination\% was also greater with SWE and Humic acid pretreatants. These results might be as an indicative of the influence of these biostmulants on stability, ion selectivity and orienting permeability membrane (Salisbury and Ross, 1992). The beneficial effects of BRS used on germination in the present investigation may be due, actually, to one or more of their effects on hormonal balance changes to favour cytokinins and auxins production so that, antioxidants production can continue when stress occurs (Schmidt, 2005) . The role of BRS used on promoting vitamin biosynthesis such as thiamine and biotein as well as their effects on activating nitrate reductance enzyme were also detected (Hatung, 2004). The hormone containing products treatments significantly improved to water status regulate cell membrane components under drought stress condition (Salisbury and Ross, 1992). 
Table (1): Mean squares from analysis of variance of sugar beet lines tested for the germination rate in filter paper and perlite medium to gather with seedling growth characters of in vitro seedling at three levels of mannitol and five BRS levels.

\begin{tabular}{|c|c|c|c|c|c|c|c|c|}
\hline \multirow{4}{*}{ Source of variance } & \multirow{4}{*}{ d.f. } & \multirow{2}{*}{\multicolumn{2}{|c|}{ Germination rate }} & \multirow{2}{*}{\multicolumn{2}{|c|}{ Fresh weight }} & \multirow{2}{*}{\multicolumn{2}{|c|}{ Dry weight }} & \multirow{3}{*}{$\begin{array}{l}\text { Root } \\
\text { length }\end{array}$} \\
\hline & & & & & & & & \\
\hline & & In paper & In perlite & $\begin{array}{c}\text { Cot.+ } \\
\text { leaves }\end{array}$ & Roots & \begin{tabular}{|c|} 
Cot.+ \\
leaves
\end{tabular} & Roots & \\
\hline & & \multicolumn{7}{|c|}{ Season 2007/2008 } \\
\hline Replication & 2 & 68.267 & & 0.000 & 0.000 & 0.000 & 0.000 & 16.544 \\
\hline & 1 & & & & & & & \\
\hline & 2 & & 282 & $90 . \varsigma$ & & & & \\
\hline BRS & 4 & 129 & & 2.236 & 0.7 & & & \\
\hline & 2 & & & & & & & \\
\hline & 4 & & & & & & & \\
\hline & 8 & 15. & 75 & 0.047 & 0.205 & & & \\
\hline l $x$ BRS & 8 & & & 0.010 & 0.004 & 0.000 & & \\
\hline \multicolumn{9}{|c|}{ Season 2008/2009 } \\
\hline & 2 & & & & & & & \\
\hline & 1 & & & 0.236 & 0.0 & & & \\
\hline Mannitol & 2 & 4059.30 & 3573.70 & 47.509 & 10.993 & 0.005 & 01 & 2607.51 \\
\hline & 4 & 1080. & $126 \varsigma$ & 2.099 & & & & \\
\hline & 2 & & & 0.019 & 0.046 & 00 & & \\
\hline Varie & 4 & 13. & 10 & 0.0 .49 & 0.003 & 0.000 & & 9.594 \\
\hline & 8 & & & 0.060 & 0.119 & 0.000 & & 20.428 \\
\hline Vriety x Mannitol x BRS & 8 & 11.90 & 10.90 & 0.007 & 0.024 & 0.000 & 0.000 & 7.144 \\
\hline
\end{tabular}

Table (2): Coefficient correlation estimated between germination rate and seedling characteristics of sugar beet .

\begin{tabular}{|l|c|c|c|c|c|c|c|}
\hline & CDW. & CFW & $\begin{array}{c}\text { Ger.In } \\
\text { perlite }\end{array}$ & $\begin{array}{c}\text { Ger.In } \\
\text { paper }\end{array}$ & RDW & RFW & RL \\
\hline CDW. & 1 & & & & & & \\
\hline CFW. & 0.929 & 1 & & & & & \\
\hline Ger.In perlite & 0.554 & 0.839 & 1 & & & & \\
\hline Ger.In paper & 0.839 & 0.929 & 0.982 & 1 & & & \\
\hline RDW & 0.982 & 0.839 & 0.643 & 0.554 & 1 & & \\
\hline RFW & 0.982 & 0.982 & 0.714 & 0.839 & 0.929 & 1 & \\
\hline RL & 0.839 & 0.982 & 0.929 & 0.982 & 0.714 & 0.929 & 1 \\
\hline
\end{tabular}

Cotyledon+leaves dry weight; CDW, Cotyledon+leaves fresh weight; CFW, Ger. Roor dry weight; RDW, root fresh weight; RFW. Root length; RL.

It has been reported that either of the BRS used increased the major elements uptake especially phosphorus and boron in addition to their effects on regulation of cell membrane components under drought stress ( Hatung, 2004 and Yan, 1993). Phosphorus have been found to be mediator of most metabolic reaction within the cell and of hormonal response in plants and accelerated germination ( El-Hadidi et al., 1981).

Data in the same tables show that there are no significant differences between presowing drought hardening seeds of the two sugar beet $\mathrm{cv}(\mathrm{s})$ treated with Ascorbic acid and those treated with either of Salsilic and Humic acids. However, the seeds of the three treatments allivated the retarding effects of mannitol on germination as compared with pretreated seeds with water (wet control). The most effective treatments was found with SWE. The effect of SWE was expected since SWE contain not only most of the major 
Helaly, M.N. et al.

and minor nutrients, amino acid and vitamins (B1, B2, C ,E) but also cytokinins, auxins, $\mathrm{GA}_{3}$ and $\mathrm{ABA}$ like substances (Hatung, 2004). Francki (1960) reported that increased trace elements; TE especially boron, B supply could explain only some of the beneficial effects of SWE. The effect of boron and TE on germination was more pronounced at drought stress than nondroughted media (Tables 3-6). The improvement of germination capacity due to boron and TE presented in SWE, especially under high level of mannitol, may be partially explained on the ground that boron and other TE may stimulat the activity of the enzyme synthesis responsible for transformation and /or translocation of carbohydrates. This may cause an increase in the osmotic pressure of the cell sap in the germinated seeds, and in the turn, could offset the osmotic unbalance between germinated seeds and the ambient solution (El-Hadidi et al., 1981). They added that boron and other TE had positive effects on sugar translocation in plant tissues. The enhancement of hydrolytic enzyme activities in the germinated seeds treated with SWE and B was previously mentioned (Salisbury and Ross, 1992). Such changes in enzyme activaties due to TE were accompanied with an intensification in respiration rate (Helely, 1972). Seaweed extract and HA may enhance hydrophobic and hydropholic antioxidant activity and thus promote germination and water status (Schmidt, 2005). The later auther added that, antioxidant status could be manipulated with exogenous application of plant growth biostimulans SWE. The increase of this antioxidants may be triggered by excess production of reactive oxygen species in the photosynthetic apparatus under stress, increased $\alpha$-tocopherol levels which may serve as an acclimation strategy of plants to tolerate water deficits.

Data in Table 3 show also that, drought stress caused by increasing mannitol level decreased significantly fresh and dry weight of the shoots (cotyledons + leaves), roots as well as whole seedling and shoot/root ratio whereas increased root length of the two $\mathrm{cv}(\mathrm{s})$ studied. The effects were more pronounced due to an increase in the osmotic pressure of mannitol level compared with that obtained at the low one. Similarly, $\operatorname{cv}(2)$ was more affected by stress than $\mathrm{cv}(1)$. The only exception was found with $\operatorname{cv}(1)$ at the low mannitol level which should an increase in all seedling characteristics studied under the investigation. Moreover, it was found that shoot growth was reduced to a much greater extent than that of the roots especially with $\operatorname{cv}(1)$; more resistance. This in turn, might account for the decrease in the shoot/root ratio. These results might be explained on the basis that , leaves seemed to be more sensitive to drought than roots. Furthermore, it might be assumed that, such phenomenon may be a kind of plant adaptation to stress which is responsible for water and essential nutritive elements uptake.

The harmfull effect of stress on germination and seedling growth represented with the dry matter accumulation seemed to be due to the suppression of plant metabolism under such condition (Demir et al., 2004) . Salisbury and Ross, 1992) reported that, drought stress adversely affect the phisco-chemical properties of the protoplasm and cell membranes. 
Table (3): Germination rate of sugar beet seeds cv(s) as affected by water stress in the presence or absence BRS under stress (mannitol) levels

\begin{tabular}{|c|c|c|c|c|c|c|c|c|c|c|c|c|c|c|c|}
\hline \multirow{4}{*}{ 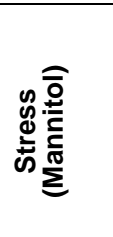 } & \multirow[b]{4}{*}{ BRS } & \multicolumn{14}{|c|}{$\begin{array}{c}\text { Germination rate } \\
\text { Season } 2007 / 2008\end{array}$} \\
\hline & & \multicolumn{7}{|c|}{ C1 } & \multicolumn{7}{|c|}{ C2 } \\
\hline & & \multicolumn{2}{|c|}{7} & \multicolumn{2}{|c|}{14} & \multicolumn{2}{|c|}{21} & \multirow[b]{2}{*}{$\sum_{\substack{\mathbb{N} \\
\Sigma}}^{\frac{0}{0}}$} & \multicolumn{2}{|c|}{7} & \multicolumn{2}{|c|}{14} & \multicolumn{2}{|c|}{21} & \multirow[b]{2}{*}{ 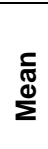 } \\
\hline & & 高 & $\frac{\stackrel{ \pm}{ \pm}}{\stackrel{ \pm}{ \pm}}$ & 高 & 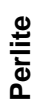 & 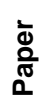 & 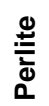 & & 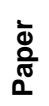 & 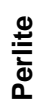 & 高 & 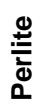 & 高 & 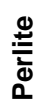 & \\
\hline \multirow{6}{*}{ Control } & Control & 30 & 35 & 60 & 62 & 63 & 65 & 52 & 28 & 32 & 58 & 60 & 62 & 65 & 51 \\
\hline & Ascorbic acid & 39 & 42 & 77 & 84 & 82 & 84 & 68 & 35 & 39 & 72 & 80 & $7 \overline{9}$ & 82 & 64 \\
\hline & Salicylic acid & 40 & 41 & 78 & 84 & 81 & 83 & 68 & 38 & 39 & 74 & 82 & 77 & 80 & 65 \\
\hline & Humic acid & 40 & 42 & 79 & 85 & 82 & 85 & 69 & 39 & 40 & 74 & 82 & 80 & 83 & 66 \\
\hline & SWE & 46 & 48 & 80 & 88 & 86 & 90 & 73 & 44 & 44 & 78 & 84 & 82 & 87 & 70 \\
\hline & Mean & .79 & $\varepsilon Y$ & Vo & $\Lambda 1$ & 19 & $\Lambda 1$ & 66 & rV & rq & VI & $\vee \wedge$ & V7 & Vq & 63 \\
\hline \multirow{6}{*}{$0.2 \mathrm{M}$} & Control & 25 & 28 & 48 & 50 & 52 & 54 & 43 & 25 & 27 & 44 & 50 & 52 & 52 & 42 \\
\hline & Ascorbic acid & 33 & 37 & 60 & 61 & 79 & 71 & 57 & 30 & 35 & 54 & 58 & 70 & 69 & 53 \\
\hline & Salicylic acid & 34 & 36 & 58 & 62 & 70 & 72 & 55 & 31 & 36 & 52 & 60 & 68 & 70 & 53 \\
\hline & Humic acid & 35 & 37 & 62 & 63 & 73 & $7 \overline{5}$ & 57 & 30 & 36 & 58 & 62 & 72 & 75 & 55 \\
\hline & SWE & 38 & 40 & 67 & 72 & 76 & 79 & 62 & 32 & 37 & 70 & 74 & 75 & 76 & 61 \\
\hline & Mean & rr & $r 4$ & 09 & $T r$ & 71 & $v$. & 55 & $r$ & ri & 44 & $\pi$ & 71 & 71 & 54 \\
\hline & Control & 20 & 22 & 42 & 44 & 48 & 50 & 38 & 20 & 23 & 42 & 44 & 45 & 50 & 37 \\
\hline & Ascorbic acid & 28 & 30 & 59 & 60 & 60 & 63 & 50 & 26 & 28 & 51 & 56 & 56 & 60 & 46 \\
\hline & Salicylic acid & 29 & 31 & 57 & 58 & 63 & 64 & 50 & 26 & 28 & 50 & 55 & 60 & 62 & 47 \\
\hline $0.3 \mathrm{M}$ & Humic acid & 32 & 34 & 55 & 57 & 65 & 66 & 51 & 30 & 31 & 51 & 56 & 62 & 62 & 49 \\
\hline & SWE & 34 & 36 & 67 & 69 & 72 & 75 & 59 & 32 & 34 & 54 & 56 & 64 & 64 & 51 \\
\hline & Mean & ra & $r \cdot$ & $0 \leqslant$ & 07 & $\pi$ & 24 & 49 & $Y V$ & Yर & or & 0. & ov & 7. & 46 \\
\hline & Control & YO & TN & 0 . & Or & $0 \leqslant$ & 09 & 44 & $T \leq$ & TV & $\leqslant \Lambda$ & 01 & Or & 07 & 43 \\
\hline & Ascorbic acid & ru & צ & 70 & 71 & $v_{1}$ & vr & 58 & r. & $r \leqslant$ & $T \leq$ & rי & $\checkmark \wedge$ & v. & 55 \\
\hline Mean & Salicylic acid & $r \leq$ & r & $7 \leq$ & 71 & vi & vr & 58 & rr & $r \leq$ & צ & $7 \leqslant$ & $\checkmark \wedge$ & $v_{1}$ & 55 \\
\hline & Humic acid & צ & rᄉ & 90 & 71 & Vr & vo & 59 & $r \mu$ & ז & 90 & 70 & Vr & VY & 57 \\
\hline & SWE & rq & $\leqslant 1$ & 71 & $\mathrm{Vr}$ & vV & $\Lambda$. & 63 & צ & rی & \ & $v_{1}$ & $v \leqslant$ & vo & 60 \\
\hline LSD at & $\begin{array}{r}\text { 5\% for Stress } \\
\text { BRS } \\
\text { StressxBRS }\end{array}$ & $\begin{array}{l}0 . \\
1 . \\
1 .\end{array}$ & 8 & & & & & & & & & & & & \\
\hline & & & & & & aso & 200 & $3 / 20$ & & & & & & & \\
\hline & Control & 33 & 34 & 59 & 60 & 65 & 66 & 53 & 30 & 31 & 60 & 62 & 64 & 67 & 52 \\
\hline & Ascorbic acid & 40 & 43 & 74 & 81 & 80 & 80 & 66 & 36 & 37 & 74 & 81 & 79 & 81 & 65 \\
\hline Control & Salicylic acid & 42 & 44 & 76 & 83 & 82 & 83 & 68 & 40 & 39 & 74 & 82 & 79 & 83 & 66 \\
\hline & Humic acid & 41 & 44 & 77 & 85 & 84 & 83 & 69 & 42 & 42 & 78 & 84 & 82 & 83 & 68 \\
\hline & SWE & 46 & 50 & 82 & 87 & 85 & 91 & 73 & 46 & 48 & 82 & 86 & 84 & 88 & 72 \\
\hline & Mean & $\leqslant$ & $\varepsilon r$ & Vई & $V \Lambda$ & $\sqrt{9}$ & $\Lambda 1$ & 66 & rq & rq & $V \leq$ & $\sqrt{9}$ & $\mathrm{vi}$ & $\Lambda$. & 65 \\
\hline & Control & 27 & 30 & 46 & 48 & 50 & 52 & 42 & 26 & 26 & 48 & 52 & 55 & 55 & 44 \\
\hline & Ascorbic acid & 34 & 37 & 58 & 58 & 57 & 70 & 5 & 33 & 32 & 58 & 58 & 72 & 67 & 53 \\
\hline $0.2 \mathrm{M}$ & Salicylic acid & 36 & 38 & 59 & 60 & 60 & 69 & 54 & 33 & 34 & 62 & 59 & 67 & 70 & 54 \\
\hline & Humic acid & 36 & 40 & 61 & 63 & 65 & 72 & 5 & 35 & 34 & 60 & 61 & 78 & 71 & 56 \\
\hline & SWE & 38 & 40 & 67 & 70 & 70 & 76 & 60 & 35 & 38 & 70 & 72 & 79 & 74 & 61 \\
\hline & Mean & $r \leq$ & rV & 01 & 01 & $\sqrt{9}$ & 71 & 56 & Tr & Tr & 7. & 7. & v. & $9 \mathrm{~V}$ & 54 \\
\hline & Control & 20 & 22 & 35 & 33 & 44 & 43 & 33 & 22 & 23 & 40 & 42 & 44 & 47 & 36 \\
\hline & Ascorbic acid & 27 & 30 & 47 & 46 & 54 & 57 & 43 & 26 & 25 & 52 & 51 & 54 & 58 & 44 \\
\hline $0.3 \mathrm{M}$ & Salicylic acid & 28 & 30 & 50 & 49 & 56 & 60 & 45 & 28 & 27 & 54 & 53 & 58 & 63 & 47 \\
\hline & Humic acid & 30 & 32 & 50 & 50 & 56 & 62 & 47 & 29 & 30 & 50 & 53 & 60 & 63 & 47 \\
\hline & & 31 & 34 & 52 & 59 & 60 & 67 & & 30 & 32 & 55 & 60 & 66 & 68 & 52 \\
\hline & Mean & YV & $r \cdot$ & $\leqslant V$ & $\leqslant V$ & $0 \leqslant$ & $0 \wedge$ & 44 & tV & YV & 0. & OY & 7. & 9. & 46 \\
\hline & Control & $r v$ & rA & $\leq V$ & $\leqslant v$ & or & $0 \leqslant$ & 43 & Y & rV & $\leq 9$ & or & • \{ & VA & 48 \\
\hline & Ascorbic acid & $r \varepsilon$ & rV & 7. & 71 & $7 \leq$ & 79 & 54 & rr & $r_{1}$ & 71 & rי & 71 & 79 & 54 \\
\hline Mean & Salicylic acid & 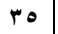 & rv & Tr & $7 \leq$ & 79 & $v_{1}$ & 5 & r & 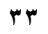 & 44 & 70 & 31 & VY & 56 \\
\hline & Humic acid & q & rq & r & Tr & 71 & Vr & & ro & ro & 4t & 79 & Vr & VY & 57 \\
\hline & & rᄉ & $\leq 1$ & $7 \mathrm{~V}$ & vi & VY & $\vee \wedge$ & 61 & $r v$ & ro & 79 & Vr & vr & $\checkmark v$ & 60 \\
\hline LSD at & $\begin{array}{c}5 \% \text { for Stress } \\
\text { BRS } \\
\text { StressxBRS }\end{array}$ & & & & & & & & & & & & & & \\
\hline
\end{tabular}


Table (4): Fresh weight (F.Wt), dry weight (D.Wt) and root length (RL) of cotyledons + leaves and roots of sugar beet $\operatorname{cv}(\mathrm{s})$ as affected by mannitol level and BRS levels.

\begin{tabular}{|c|c|c|c|c|c|c|c|c|c|c|c|}
\hline \multirow{5}{*}{ 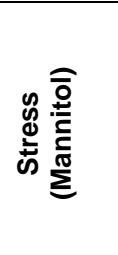 } & \multirow{5}{*}{ BRS } & \multicolumn{10}{|c|}{ Seedling characters } \\
\hline & & \multicolumn{10}{|c|}{ Season $2007 / 2008$} \\
\hline & & \multicolumn{5}{|c|}{ C1 } & \multicolumn{5}{|c|}{ C2 } \\
\hline & & \multicolumn{2}{|c|}{ F.Wt g } & \multicolumn{2}{|c|}{ D.Wt g } & \multirow[b]{2}{*}{$\begin{array}{l}\underline{E} \\
\overrightarrow{0} \\
\underline{x}\end{array}$} & \multicolumn{2}{|c|}{ F.Wt g } & \multicolumn{2}{|c|}{ D.Wt g } & \multirow[b]{2}{*}{ 조 등 } \\
\hline & & 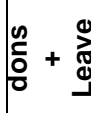 & 号 & 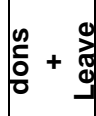 & $\begin{array}{l}\stackrel{n}{0} \\
\stackrel{0}{0}\end{array}$ & & 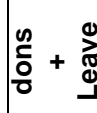 & 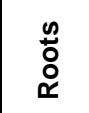 & $\mid \begin{array}{l}\mathscr{c} \\
\frac{\mathrm{O}}{0}\end{array}$ & $\begin{array}{l}\stackrel{n}{\circ} \\
\stackrel{0}{0}\end{array}$ & \\
\hline \multirow{6}{*}{ Control } & Control & 4.266 & 1.980 & 0.043 & 0.020 & 55 & 4.350 & 2.005 & 0.044 & 0.020 & 56 \\
\hline & Ascorbic acid & 4.601 & 2.308 & 0.046 & 0.023 & 58 & 4.702 & 2.335 & 0.048 & 0.022 & 58 \\
\hline & Salicylic acid & 4.514 & 2.316 & 0.045 & 0.023 & 63 & 4.713 & 2.410 & 0.048 & 0.024 & 64 \\
\hline & Humic acid & 4.882 & 2.708 & 0.049 & 0.027 & 67 & 4.840 & 2.650 & 0.050 & 0.025 & 65 \\
\hline & SWE & 5.302 & 3.082 & 0.053 & 0.031 & 72 & 5.297 & 3.104 & 0.053 & 0.030 & 74 \\
\hline & Mean & 4.713 & 2.475 & 0.047 & 0.025 & 63.0 & 4.780 & 2.501 & 0.049 & 0.024 & 63.4 \\
\hline \multirow{6}{*}{$0.2 \mathrm{M}$} & Control & 3.000 & 1.442 & 0.030 & 0.014 & 50 & 3.080 & 1.508 & 0.031 & 0.015 & 53 \\
\hline & Ascorbic acid & 3.413 & 1.508 & 0.034 & 0.015 & 52 & 3.410 & 1.714 & 0.034 & 0.017 & 56 \\
\hline & Salicylic acid & 3.384 & 1.565 & 0.034 & 0.016 & 53 & 3.545 & 1.690 & 0.036 & 0.017 & 56 \\
\hline & Humic acid & 3.602 & 1.675 & 0.035 & 0.017 & 55 & 3.600 & 1.712 & 0.036 & 0.017 & 58 \\
\hline & SWE & 4.211 & 1.800 & 0.043 & 0.018 & 58 & 4.180 & 2.002 & 0.042 & 0.020 & 60 \\
\hline & Mean & 3.522 & 1.598 & 0.035 & 0.016 & 53.6 & 3.563 & 1.725 & 0.036 & 0.017 & 56.5 \\
\hline \multirow{6}{*}{$0.3 \mathrm{M}$} & Control & 1.810 & 1.118 & 0.018 & 0.011 & 41 & 2.005 & 1.007 & 0.020 & 0.010 & 44 \\
\hline & Ascorbic acid & 2.008 & 1.140 & 0.020 & 0.012 & 44 & 2.312 & 1.156 & 0.022 & 0.012 & 46 \\
\hline & Salicylic acid & 2.214 & 1.155 & 0.022 & 0.012 & 43 & 2.368 & 1.150 & 0.024 & 0.012 & 48 \\
\hline & Humic acid & 2.405 & 1.170 & 0.024 & 0.012 & 44 & 2.414 & 1.178 & 0.024 & 0.012 & 50 \\
\hline & SWE & 2.708 & 1.240 & 0.027 & 0.013 & 46 & 2.600 & 1.198 & 0.026 & 0.012 & 50 \\
\hline & Mean & 2.229 & 1.165 & 0.022 & 0.012 & 43.6 & 2.340 & 1.138 & 0.023 & 0.012 & 47.6 \\
\hline \multirow{4}{*}{ Mean } & Control & $\begin{array}{l}3.025 \\
3.341\end{array}$ & $\begin{array}{l}1.513 \\
1.652\end{array}$ & 0.030 & 0.015 & 48.7 & 3.145 & 1.507 & 0.032 & 0.014 & 51.0 \\
\hline & Salicylic acid & $\begin{array}{l}3.341 \\
3.371\end{array}$ & $\begin{array}{l}1.652 \\
1.679\end{array}$ & $\begin{array}{l}0.033 \\
0.034\end{array}$ & $\left|\begin{array}{l}0.017 \\
0.017\end{array}\right|$ & $\begin{array}{l}51.3 \\
53.0\end{array}$ & $\begin{array}{l}3.475 \\
3.542\end{array}$ & $\begin{array}{l}1.735 \\
1.750\end{array}$ & $\begin{array}{l}0.035 \\
0.036\end{array}$ & $\begin{array}{l}0.017 \\
0.018\end{array} \mid$ & $\begin{array}{l}53.3 \\
55.9\end{array}$ \\
\hline & Humic acid & 3.360 & 1.851 & 0.036 & 0.019 & 55.3 & 3.618 & 1.847 & 0.037 & 0.019 & 57.7 \\
\hline & SWE & & & 0.041 & 0.021 & 58.7 & 4.026 & 2.101 & 0.040 & 0.020 & 61.3 \\
\hline LSD a & 5\% for Stress & 0.001 & 0.002 & 0.0003 & 0.0001 & 0.14 & 0.001 & 0.003 & 0.0003 & 0.000 & 0.11 \\
\hline & BRS & 0.001 & 0.002 & 0.0003 & 002 & 0.19 & 0.002 & 0.004 & 0.0003 & 0.000 & 0.14 \\
\hline & StressxBRS & & & 0.0005 & 003 & & 0.002 & & & & 0.24 \\
\hline & & & & Sea & ason 20 & $008 / 20$ & 009 & & & & \\
\hline & Control & 4.260 & 1.974 & 0.043 & 0.021 & 57 & 4.344 & 2.000 & 0.044 & 0.020 & 56 \\
\hline & Ascorbic acid & 4.680 & 2.319 & 0.047 & 0.023 & 63 & 4.780 & 2.345 & 0.048 & 0.024 & 58 \\
\hline Control & Salicylic acid & 4.574 & 2.322 & 0.046 & 0.023 & 65 & 4.745 & 2.400 & 0.048 & 0.024 & 64 \\
\hline & Humic acid & 4.845 & 2.544 & 0.049 & 0.027 & 65 & 4.860 & 2.453 & 0.049 & 0.025 & 65 \\
\hline & \begin{tabular}{|l} 
SWE \\
\end{tabular} & 5.333 & 3.100 & 0.054 & 0.031 & 70 & 5.245 & 2.824 & 0.053 & 0.027 & 74 \\
\hline & Mean & 4.738 & 2.450 & 0.048 & 0.025 & $7 \leq, 0$ & 4.795 & 2.404 & 0.048 & 0.023 & $74, \varepsilon$ \\
\hline & Control & 3.041 & 1.398 & 0.031 & 0.014 & 53 & 3.112 & 1.418 & 0.031 & 0.013 & 53 \\
\hline & Ascorbic acid & 3.225 & 1.700 & 0.033 & 0.017 & 58 & 3.440 & 1.722 & 0.035 & 0.015 & 56 \\
\hline $0.2 \mathrm{IVI}$ & Salicylic acid & 3.372 & 1.700 & 0.034 & 0.017 & 55 & 3.509 & 1.745 & 0.035 & 0.016 & 56 \\
\hline & Humic acid & 3.514 & 1.662 & 0.035 & 0.016 & 56 & 3.585 & 1.787 & 0.036 & 0.015 & 58 \\
\hline & SWE & 4.198 & 1.745 & 0.042 & 0.018 & 62 & 4.171 & 2.018 & 0.042 & 0.016 & 60 \\
\hline & Mean & 3.470 & 1.641 & 0.035 & 0.016 & $07, \Lambda$ & 3.563 & 1.738 & 0.036 & 0.015 & 04,7 \\
\hline & Control & 1.714 & 1.110 & 0.017 & 0.011 & 40 & 2.011 & 1.002 & 0.020 & 0.010 & 42 \\
\hline & Ascorbic acid & 2.000 & 1.224 & 0.020 & 0.012 & 45 & 2.319 & 1.244 & 0.023 & 0.011 & 45 \\
\hline $0.3 \mathrm{M}$ & Salicylic acid & 2.208 & 1.250 & 0.022 & 0.013 & 45 & 2.350 & 1.256 & 0.024 & 0.011 & 45 \\
\hline & Humic acid & 2.312 & 1.274 & 0.023 & 0.013 & 44 & 2.400 & 1.255 & 0.024 & 0.012 & 47 \\
\hline & SWE & 2.622 & 1.354 & 0.027 & 0.014 & 50 & 2.565 & 1.300 & 0.026 & 0.013 & 49 \\
\hline & Mean & 2.171 & 1.242 & 0.022 & 0.013 & $\varepsilon \varepsilon, \lambda$ & 2.328 & 1.211 & 0.023 & 0.012 & $\leqslant 0, V$ \\
\hline & Control & 3.005 & 1.491 & 0.030 & 0.015 & 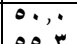 & 3.155 & 1.473 & 0.032 & 0.014 & 0,4 \\
\hline & Ascorbic acid & 3.302 & 1.748 & 0.033 & 0.017 & $00, r$ & 3.513 & 1.770 & 0.035 & 0.016 & or, \\
\hline Mean & Salicylic acid & 3.385 & 1.757 & 0.034 & 0.018 & $00, \cdot$ & 3.535 & 1.800 & 0.036 & 0.016 & $00,$. \\
\hline & Humic acid & 3.557 & 1.827 & 0.036 & 0.019 & $\Delta 0, \cdot$ & 3.615 & 1.832 & 0.036 & 0.017 & 07,7 \\
\hline & SWE & & 2.066 & & & $7 \cdot, 7$ & & 2.047 & 0.040 & 0.020 & 71,1 \\
\hline LSD at & $\begin{array}{c}\% \text { for Stress } \\
\text { BRS } \\
\text { StressxBRS }\end{array}$ & $\begin{array}{l}0.001 \\
0.001 \\
0.002\end{array}$ & $\begin{array}{l}0.001 \\
0.001 \\
0.002\end{array}$ & \begin{tabular}{|l|}
0.000 \\
0.000 \\
0.000
\end{tabular} & $\begin{array}{l}0.0001 \\
0.0002 \\
0.0003\end{array}$ & $\begin{array}{l}0.18 \\
0.22 \\
0.46\end{array}$ & $\begin{array}{l}0.001 \\
0.001 \\
0.001\end{array}$ & $\begin{array}{l}0.001 \\
0.002 \\
0.002\end{array}$ & \begin{tabular}{|l|}
0.000 \\
0.000 \\
0.000
\end{tabular} & \begin{tabular}{|l|}
0.000 \\
0.000 \\
0.0002
\end{tabular} & $\begin{array}{l}0.24 \\
0.33 \\
0.47\end{array}$ \\
\hline
\end{tabular}


Inhibition of cytokinin biosynthesis and hormonal unbalces, water content, some plant nutrient uptake, antioxidant enzymes (SOD, GR, ASP) , biosynthesis of $\alpha$-tocopherol, ascorbic acid and carotenoids as well as net photosynthetic rate ccompanied with high respiration rate were also reported under stress condition (Schmidt, 2005).

Soaking sugar beet seeds with each of the BSR used can alleviate the harmful effects of drought stress on all seedling characters studied (Tables 3and 4). It can be noticed that, soaking seeds in SWE , HA, Ascorbic and Salsilic acids in a descending order counteracted the depressing effects of drought stress on seedling growth to a different extent. It may be suggested that sugar beet seedling especially with $\operatorname{cv}(2)$ subjected to these treatments acquired a reasonable drought tolerance capacity. The more effective treatment restoring most of the plant growth capacity under high mannitol level was the biostimulants SWE and HA. This might be attributed to its effects on activate root cells and stimulate biosynthesis of endogenous cytokinins from the roots (Schmidt, 2005). The results in the present investigation indicate that soaking seeds in antioxidants used should be considered on soaking the factors which may improve growth in mannitol affected seedling. It has been show that provision for adequate uptake of water and nutrients or adjusting the hormonal balance within the plant tissues were essential for improving development at of sugar beet under stress condition (Demir et al., 2004). Results in the same tables show also that the effect of decreasing water potential in 0.2 and $0.3 \mathrm{M}$ mannitol levels resulted in seedling with less fresh weight during the germination processes and early growth compared with the control.

Analysis of variance for fresh weight of cotyledons and leaves as well as roots indicated that stress condition and BRS had significant differences for these parameters. Stress decreased water content and assimilate accumulation in the seedling of the two $\mathrm{cv}(\mathrm{s})$ as a consequence of osmotic pressure induced by mannitol.

The interaction effects between BRS and mannitol stress were significants for the germination rate pointing to the fact that differences in BRS type affecting the germination response of sugar beet are expressed at early stages under specific stress condition. The differences in germination \% of seeds subjected to stress levels were more detectable in SWE and HA. Germination of seeds with ascorbic and salysilic acids proved to be most sensitive to water restriction at the higher level of osmotic potential but with HA revealed a relatively stable tolerance in both stress levels.

Evaluation of seedling characters presented as germination \% of the control, data show a decrease for all growth parameters as stress levels intensified. The most tolerant seedling showed a better biological efficiency (increase in size and weight) under increased water deficiency, while a different distribution of biomass in leaves and roots were noted. Here again, SWE and HA responded best for relative germination and relative growth of root length. The other various BRS used responded differently for the relative growth measured as fresh weight. A positive correlation was found between germination rates and seedling characters in most cases except for the \% of abnormal seedling recorded under high stress level without BRS addition. 
Moreover, Seed germination was closely related to the root length and in severe stress condition the highest value of root length was allocated to the drought - resistance. Absolute increases in root elongation rate are strongly related to a high water statues in the plant organs. The exponents of Bewley and Black (1985) performed on Brassica oleracea var stalica seeds in water stress medium demonstrated that, the sensitively of radical expansion and radical growth to water stress is markedly different. There are a distinguish between seed germination which is completed when the radical expand and penetrates the medium, consisting of only cell elongation and the cell division and radical (root length) which starts later on. On sugar beet, Sadeghian and Yavari (2004) indicated that the stress levels exceeding -8 bar did not permit seed imbibitions and distinction among the seed lines. Similarly, germination \% of Brassica oleracea seeds was decline as water stress increase from 0 to -8 bar and finally stopped at -14 bar, whereas radical growth was started at -8 bar water potential, declined at -16 bar and slopped at -22 bar (Bewley and Black,1985) .

Distinction of significant differences in sugar beet seedling growth and physiological performance in water restriction stress leed to the conclusion that these parameters, especially germination rate and seedling root length, could be used as a criteria in screening the most tolerant progeny lines against abiotic stresses. In vitro controlled conditions seems to be more amenable for evaluating of genetic materials at early growth phase. Progeny lines having stable germination and seedling growth properties against a range of induced osmotic pressure may then be included in breeding programmes for yield potential and stability under water- restricted condition in experimental plot.

\section{Effect of BRS with or without stress on $\mathrm{H}_{2} \mathrm{O}_{2}$, AsA , Glutathione, Proline and the activity of antioxidant enzyme:}

Data in Table 5, show that, Increasing mannitol level was associated with a marked reduction in AsA, glutathione; GSH, catalase;CAT, guaiacol peroxidase;GPOD and superoxide dismutase;SOD whereas, increased that of concentrations of $\mathrm{H}_{2} \mathrm{O}_{2}$ and proline of the shoots in both seedling $\mathrm{cv}(\mathrm{s})$. The concentrations of $\mathrm{H}_{2} \mathrm{O}_{2}$ and proline were decreased due to BRS application. The decrease was more pronounced in response to SWE treatment in both $\mathrm{cv}(\mathrm{s})$ of sugar beet. The interaction treatments show that, the antioxidants counteracted the depressing effects of mannitol on all parameters studied in both $\mathrm{cv}(\mathrm{s})$ and $\mathrm{cv}(2)$ showed, in general, high concentration of $\mathrm{H}_{2} \mathrm{O}_{2}$ and proline values compared with cV (1).

A specific roles of BRS on elevating stress were reported by Metwally, Reda (2009) who reported that, the alleviation effect of ascorbic acid may be due to its enhancing effect on cell division and synthesis of hydroxyl-proline-rich protein. Wingate et al., (1988) found that, antioxidants regulated the gene expression and being the precursor of phytochelatins. Increasing $\mathrm{H}_{2} \mathrm{O}_{2}$ under drought stress as well as other reductive oxygen species; antioxidants leading to oxidative stress is a principal component of their damaging effect on plant tissues (Schutzendubel and Polle, 2002).The protective role of antioxidants against $\mathrm{H}_{2} \mathrm{O}_{2}$ accumulation was previously reported (Cheng, 2003 and Munne- Bosch, 2005). 
Table (5): Effects of BRS with or without mannitol level on $\mathrm{H}_{2} \mathrm{O}_{2}\left(\mu \mathrm{g} / \mathrm{g}^{-1}\right.$ F.wt), AsA ( $\mu \mathrm{g} / \mathrm{g}^{-1}$ F.wt), glutathione;GSH ( $\mu \mathrm{g} / \mathrm{g}^{-1}$ F.wt), proline ( $\mathrm{mg} / \mathrm{g}^{-1}$ F.wt.)and the activity of antioxidant enzyme $\left(\mu \mathrm{g} / \mathrm{g}^{-1} \mathrm{~F}\right.$.wt)of shoots of sugar beet $\mathrm{cv}(\mathrm{s})$ during the growing seasons of 2008/2009.

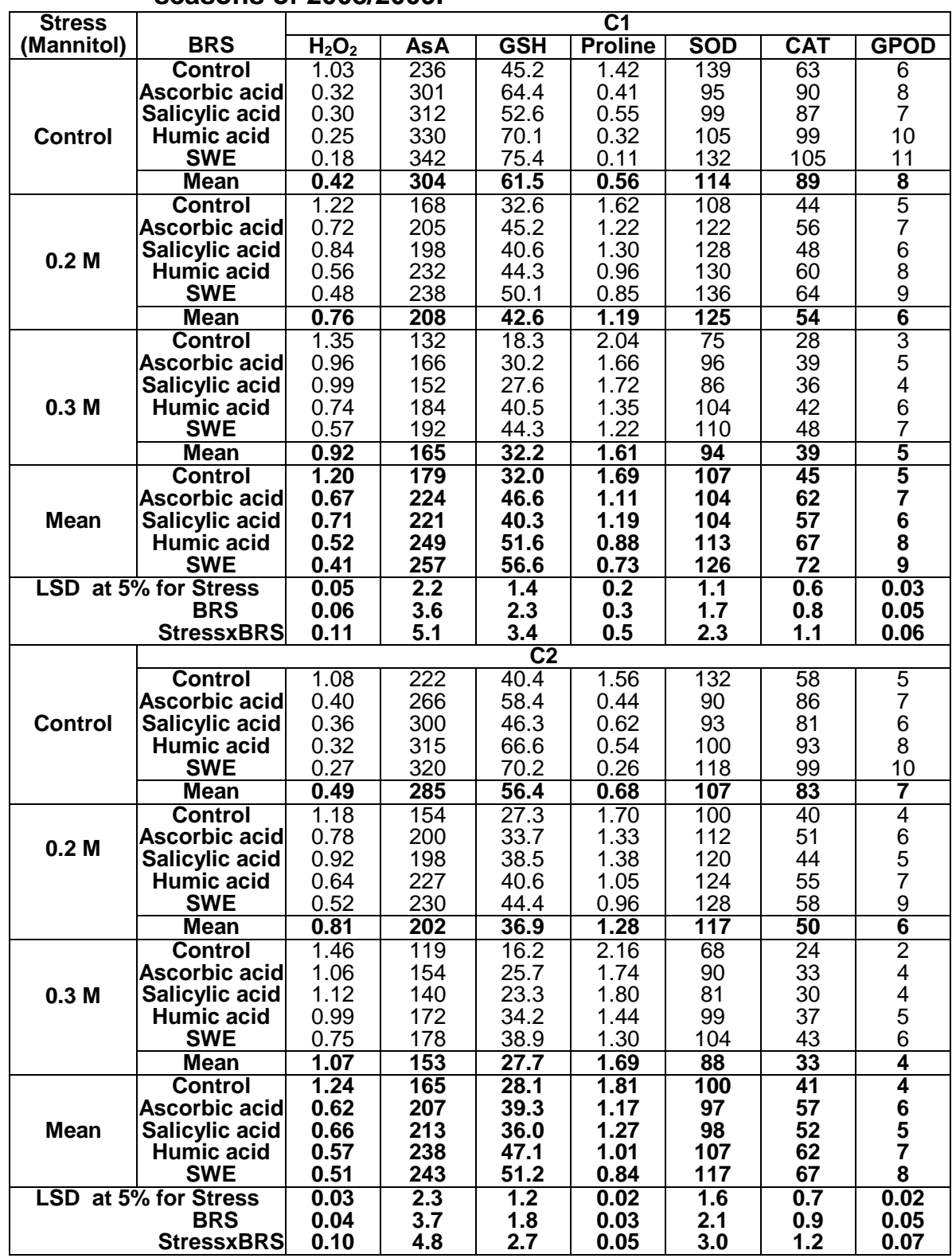

According to Abdel-Salam, Heba (2006) , AsA reduced glutathione; GSH and $\alpha$ - tocopherol; T have each been shown to act as antioxidants in the detoxification of reactive oxygen species; ROS in ascorbic cells. They 
have central and interrelated roles acting both chemically and as substrates in enzyme- catalyzed detoxification reactions. AsA is an important compound of the plant antioxidant defence system and serves as a reductant for the peroxidative removal of $\mathrm{H}_{2} \mathrm{O}_{2}$. reduced glutathione; $\mathrm{GSH}$ directly reduces most of reactive oxygen species; ROS and maintain the accorbate pool in plant cell. The reduction effects of AOs on proline concentration may be due to their stress alleviation effects (Abdel-Salam, Heba 2006) in addition to their enhancing effects on cell division (Sanchez-Fernandez, et al., 1997) These effects leed to an acceleration of proline consumption in the synthesis of hydroxyl-proline-rich proteins which are necessary for progression through the cell cycle (Arrigoni et al., 1992).

Data tablueted in Table 5 show also that, BRS especially with SWE increased the concentrations of AsA and GSH in sugar beet seedlings of both Cv (s) grown under water stress used. Moreover, SWE showed higher effects than that of HA or AsA or SIA on increasing enzymatic and non-enzymatic antioxidants concentrations in both cV (s) since treatment SWE showed highest values in this respect.

The interaction effects between the $\mathrm{cv}$ (s), stress and the BRS treatments on antioxidants enzymes activates were significant. These results indicated that, BRS induced oxidative stress. Smeets et al., (2005) reported that, plant cell respond to elevated levels of oxidative stress by activating their antioxidative defence system and the first group of enzymes involved in this defence are the ROS- quenching enzymes such as; CAT, PODS and SOD. Cheng (2003) ,reported that, CAT, PODS and SOD are important enzymes for plant adaptation to environmental stresses as the harmonious of the three enzymes make the balance between ROS production and elimination, thus keeping the level of ROS in plant tissues low, to prevent the injury of cells. In peroxisomes, $\mathrm{H}_{2} \mathrm{O}_{2}$ can be destroyed by CAT. CAT produces molecular oxygen and water from two molecules of $\mathrm{H}_{2} \mathrm{O}_{2}$. Since these two molecules must impinge simultaneously at the active site, CAT has a very high maximum velocity. PODS is an important role in scavenging $\mathrm{H}_{2} \mathrm{O}_{2}$ and organic peroxidase (Smeets et al., 2005) . Moreover, Abdel-Salam, Heba (2006) reported that, SOD causes the catalytic dismutation of potentially toxic superoxide anion radical $\left(\mathrm{O}^{2-}\right)$ to $\mathrm{H}_{2} \mathrm{O}_{2}$ whereas, CAT decomposes $\mathrm{H}_{2} \mathrm{O}_{2}$ to water and oxygen molecule, both enzymes provide an efficient mechanism for the removal of free radicals from the cells.

Total carbohydrates, total nitrogen, total soluble solids and osmotic pressure:

The data representing total carbohydrates, total nitrogen, total soluble solids and osmotic pressure of the two sugar beet cv (s) as affected by certain Biostimulant Remediation Substances; BRS under mannitol stress condition are presented in table 6 .

Results in Table (6) revealed that increasing mannitol level was associated with a marked reduction in total carbohydrates and total $\mathrm{N}$ concentrations, whereas, increased that of T.S.S. and osmotic pressure of the shoots in both $\mathrm{cv}(\mathrm{s})$ tended to increase. The limitation of carbohydrates production under stress condition may be due to one or another of the two ways: first; an adjustment in the internal osmotic pressure of the cell sap as a 
trial to go along with the external drought media(Salisbury and Ross, 1992) or increasing the intermediate substances of organic products (Hatung, 2004). These organic substances may serve as acceptor for the inorganic one , particularly those of $\mathrm{N}, \mathrm{P}, \mathrm{K}, \mathrm{Mg}, \mathrm{Ca}, \mathrm{Zn}, \mathrm{Fe}$ and $\mathrm{Cu}$, thus causing an increase in the inorganic substances at the expense of total $\mathrm{N}$ ( Crouch and Van Staden, 1993). Second: a production of higher energy, by means of respiration in order to overcome the relatively low availability condition of water and nutritional elements in stressed media ( Demir et al., 2004). Since carbohydrates are the principal substances used in respiration a depression in carbohydrates content of plants growth under stress condition could be expected. Other possibility could be the suffering of plants growth on stress media from K deficiency (Salisbury and Ross, 1992), which in turn may results in carbohydrates diminish. El-Hadidi, et al., (1981) suggested that $\mathrm{N}$ compounds under stress may not be fully utilized and consequently the accumulation of these substances is more rapid than their utilization for the formation of new cells and tissues. The increase in total $\mathrm{N}$ of sugar beet under stress condition noticed in the present investigation in connection with the highly depressed in leaves growth more than in roots could be explained on the basis that $\mathrm{N}$ uptake was not affected as leaves growth by mannitol increase and consequently $\mathrm{N}$ accumulation was affected .

Regarding the effects of pretreatment with BRS, it was found that soaking the two $\operatorname{cv}(\mathrm{s})$ of sugar beet in SWE, HA, AsA and SA increased in a descending order the \% of total carbohydrates in the two plant $\mathrm{cv}(\mathrm{s})$. Moreover, BRS treatments showed an additive

effects to that of stress on protein $-\mathrm{N}$ and total nitrogen concentrations as well as total soluble solids and osmotic pressure. However, soluble $\mathrm{N}$ showed a decrease as a result of pretreatant seeds with each of the BRS used. The increase in protein and total $\mathrm{N}$ may be attributed to a corresponding increase in amino acids and nucleic acids intimately connected with and incorporated into nitrogenous compounds (O-Donnell, 1973).

Data in the present investigation indicated that, the effects of these substances were more pronounced under high mannitol level. In this context, it was found that hardening treatments with biostimulants could partially overcome the disturbances occurred in the physiological statues due to stress condition. The pretreated seeds restored growth, increased chlorophylls biosynthesis (Gardaye and Churin, 1996), inhibited the activity of free radical groups which are major elements for chlorophyll degradation (Fletcher et al., 1988), stimulated the biosynthesais of $\alpha$ - tocopherol, ascorbic acid and carotenoids in the chloroplast which protect photosynthetic apparatus of PSII (Hatung, 2004), promoted the accumulation of reducing sugars which increased wilting resistance through enhancing osmotic pressure inside the plant tissues (Salisbury and Ross, 1992) and stimulated the chloroplast development ( Demir et al., 2004) . Moreover, it was reported that, BRS regulate the hormonal balance, water status and nutrient availability within the plant tissues (Demir et al., 2004). Likewise, Schmidt, (2005)found that SWE increased endogenous cytokinins biosynthesis under drought stress which regulate nutrient absorption cell membrane components as well as, redistribution and determination of the overall ions uptake selectivities 
Table (6): Total carbohydrates, total nitrogen, total soluble solids and osmotic pressure(O.P) of the two sugar beet cv (s) as affected by certain Biostimulant Remediation Substances; BRS under mannitol stress condition during the two growing seasons of 2007/2008 and 2008/2009.

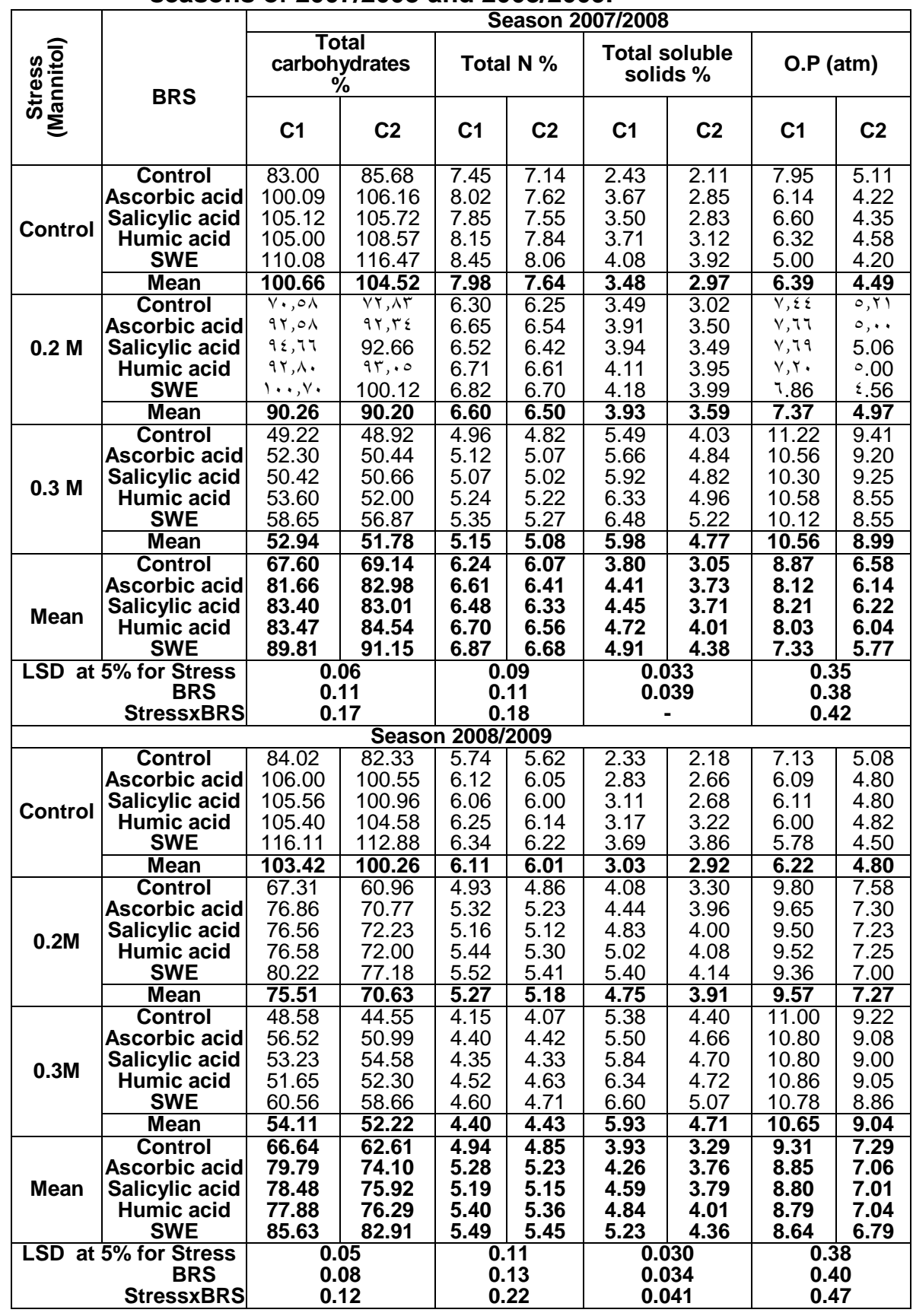




\section{REFERENCES}

A.O.A.C., Association of Official Analytical Chemists. (2000). Official Methods of Analysis. Association of Official Agriculture Chemists. $17^{\text {th }}$ Ed. PPub. Washington. DC., USA.

Abd El-Salam, Heba M.I. (2006). Phytochemical studies on certain fabaceous plants grown under heavy metal stress. Ph. D. Thesis, Fac. of Agric. Mansoura Univ., Egypt.

Arrigoni, O; L. De Gara; F. Tommasi and R. Liso (1992). Changes in the ascorbic system during seed development of Visia faba L. Plant Physiol., 99: 235-238.

Bates, L.S.; R.P. Waldren and I.D. Teare (1973). Rapid determination of free praline for water-stress studied. Plant and Soil, 39: 205-207.

Bewley, J.D. and M.Black (1985). Seeds: Physiology of development germination. Pp. 124-125. Plenum Publishing Corporation, NY.

Borsani, O.; V.Valpuesta and M.A.Botella (2001). Evidence for a role of salicylic acid in the oxidative damage generated by nacl and osmotic stress in Arabidopsis seedlings. Plant Physiol. 126: 1024-1030.

Cakmak, I. and H.Marschner, (1992). Magnesium deficiency and high light intensity enhance activities of superoxide dismutase, Ascorbate peroxidase and glutathione reductase in been leaves. Plant Physiol., 98: 1222-1227.

Chattopadhyay, MK.; BS.Tiwari; G. Chattopadhyay; A. Bose; DN. Sengupta; B. Ghosh (2002). Protective role of exogenous polyamines on salinitystressed rice (Oryza sativa) plants. Physiol.plant, 116: 192-199.

Cheng, S. 2003. Effects of heavy metals on plants and resistance mechanisms. Environ. Sci. Pollut. Res., 10: 256-264.

Clapp, C.E.; R.S.Vial;Y. Chen; A.J.Palazzo; V.W.Cline; J.M.Baker ( 2002). Stimulation of plant growth by humic substances. ASA-CSSA-SSSA Annual Meeting Abstracts. Paper No. S03-clapp 125043-Poster .

Crouch, I . J., and J. Van-Staden (1993). Evidence for the presence of plant growth regulators in commercial seaweed products. Plant Growth Regul. 13: 2129.

Demir, D., A. Günes, A. Inal and M. Alpaslan (2004). Effects of humic acids on the yield and mineral nutrition of cucumber (cucumis sativus I.) grown with different salinity levels.ishs acta horticulturae 492(2004).

El-Hadidi, M.S.; M.N.M. Helaly and M.N. El-Abaseri (1981). Effect of cycocel and kinetin on growth and metabolic processes in tomato plants (Lycopersicon esculentum, L.) grown under saline conditions Res. Bull., 438, Fac. Agric. Zagazig Univ., Egypt.

El-Hosieny, Hanan, M.R. (2008). Comparative physiological and anatomical studies on somaclonal varieties of date palm (Phoenix dactylifera, L.) tissue culture. Ph.D Thesis, Fac. of Agric. Mansoura Univ., Egypt.

Faust, R.F. (2006). Humate and Humic Acids for Agriculture Users. Guide". Monty;s Plant Food Substances htm. 
Fletcher, R.A., G. Hofstra and J. Gao (1988). Comparative fungitoxic and plant growth regulating properties of triazole derivatives. Plant Cell Physiology 27:367-371.

Francki, R.I.B. (1960). Manurial value of seaweeds: I. Effects of pachymenia himantophora and durvillea antartica meals on plant growth. Plant and Soil 12:297-310.

Garbaye, J. and J.L. Churin (1996). Effect of ectomycorrhizal inoculation at planting on growth and foliage quality of Tilia tomentosa. J.Arboric. 22(1):29-33.

Ghoulam, C. and K. Fares (2001). Effect of salinity on seed germination and early seedling growth of sugar beet (Beta vulgaris L.). seed Sci. Tochnol. 29, 357-364.

Hatung, W. (2004). Plant response to stress: Abscisic acid fluxes. Marcel Dekker Inc., New York. pp. 540-690.

Helaly, M.N.M. (1972). Studies on the effects of salinity on germination, growth and chemical composition of Egyptian henbane (Hyoscyamus muticus, L.). M. Sc. Thesis, Faculty of Agric. Cairo Univ., Egypt.

Hoagland, D.R. and D.I. Arnon, (1950). The water culture method for growing plants without soil. California Exp. Stat. Circular No. 347. The College of Agric. Univ. Calif. Berkelen, CA.

Metwally, Reda S. A. (2009). Physiological studies on the effect of some antioxidants on sweet pepper plant under salinity stress. Ph.D. Thesis, Fac. of Agric. Mansoura Univ., Egypt.

Mishra, N. M.; K. Makkar and S. Verma (2003). Polyamines in Plant Growth and Development. In: A. Hemantranjan, Editor, Advances in Plant Physiology, Scienctific Publishers, India (2003), pp. 155-224.

Munne-Bosch, S. (2005). The role of a-tocopherol in plant stress. J. Plant Physiol., 162: 743-748.

Nabati, D. A., R.E. Schmidt and D.J. Parrish (2005). Alleviation of salinity stress in Kentucky bluegrass by plant growth regulators and iron. Crop Science 34:198-202.

Nour, T.A. (1971). Effect of saline substrate on the photosynthetic apparatus , growth and some metabolic change of wheate plants. M.Sc. Thesis Faculty of Agric. Ain Shams Univ., Egypt.

O'Donnell, R.W. (1973). The auxin-like effects of humic preparations from leonardite. Soil Sci. 116:106-112.

Ozdoba, (2006). Effects of Lactate, Humate, and Bacillus Subtilis on the Growth of Tomato Plants in Hydroponic Systems Leonardite and Humified Organic Matter" ( D.M Ozdoba et. al., Luscar Specialty Products Division).

Panda, S.K. and M.H.Khan (2003). Salt stress influences lipid peroxidation and antioxidants in the leaf of an indica rice (Oryza sativa L.). Physiology-and-Molecular-Biology-of-Plants, 9: 2, 273-278.

Sadeghian, S.Y. and N. Yavari (2004). Effect of water deficit stress on germination and early seedling growth in sugar beet . J. Agronomy \& Crop Science 190, 138-144. 
Salisbury, F.B. and C.W. Ross, (1992). Environmental physiology. In: Plant Physiology, $4^{\text {th }}$ ed), p. 449-500. Wadsworth Pub. Company, Beimont, CA, USA.

Sanchez-fernandez, R.; M. fricker; L-B Corben; N.S. White ; N. Sheard; C.J. Leaver; M. Van Montagu ; D. Inze and M.J. May (1997). Cell proliferation and hair tipgrowth in the Arabidopsis root are under mechanistically different forms of redox control. Proc. Natl. acad. Sci. U.S.A. , 94: 2745-2750.

Schmidt R.E. ( 2005). Biostimulants function in turfgrass nutrition. Phd emeritus virginia tech.

Schutzendubel, A. and A. Polle (2002). Plant response to a biotic stress:heavy metal-induced oxidative stress and protection by mycorrhization. J. Exp. Bot., 53: 1351-1365.

Slatyer, R.U. and J.C. Mcllory (1961). Practical micromatology (with special reference to the water factor in soil-plant atmosphere relationships) UNESCO.

Smirnoff, N. (1995). Antioxidant systems and plant response to the environment. In N. Smirnoff (Ed.), Environment and plant metabolism: Flexibility and acclimation, pp.217-243. Oxford, UK: BIOS Scientific Publishers Ltd.

Smith, S.E. and A.K. Doberenz (1987). Seed age and salt tolerance at germination in Alfalfa. Crop Sci. 27, 1053-1056.

Tang, W. and R.Newton (2005). Polyamine reduced salt induced oxidative damage.Plant growth Regulation.46: 31-43.

Tugnoli, D.V. and D.G. Bettini (2001). Verifying the germinability of commercial sugar beet seeds under laboratory conditions and from emergence in the field. Proceedings of the $46^{\text {th }}$ IIRB Congress, 26-27 June, Bruges, pp. 333-340.

Urbanek, H. ; E. Kuzniak -Gebarowska, and H. Herka (1991). Elicitation of defense responses in been leaves by Botrytis cinerea polygalacturonase. Act Physiol. Plant., 13: 42-50.

Van Rossun, M.W.P.C.; M. Alberda and L.H.W. Van Der Plas (1997). Role of oxidative damagr in tulip scale micropropagation. Plant Sci., 130: 207216.

Velikova, V. ; I. Yordanov and A. Edreva (2000). Oxidative stress and some antioxidant system in acid rain-treated been plants protective role of exogenous polyamines. Plant Sci., 151: 59-66.

Wingate, V.P.M. ; M.A. Lawton and C.J. lamb (1988). Glutathione causes a massive and selective induced of plant defense genes. Physiol. Plant,31: 205-211.

Yan, J. (1993). Influence of plant growth regulators on turfgrass polar lipid composition, tolerance to drought and saline stresses, and nutrient efficiency. Ph.D.. Dissertation. CSES, Virginia Tech.

Zhao F.G. and Qin, P. (2004). Protective effect of exogenous polyamines on root tonoplast function against salt stress in barley seedlings. Plant Growth Regulation 97-103, (2004). 
Helaly, M.N. et al.

تأثير بعض المواد المعالجة الحيوية على إنبات ونمو البادرة لبعض أصناف بنجر السكر تحت ظروف الآجهاد.

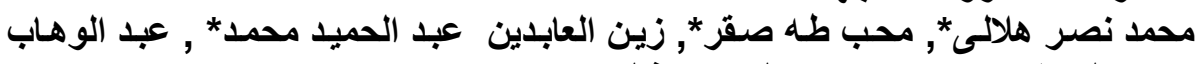

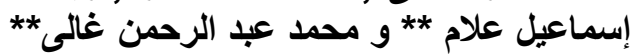

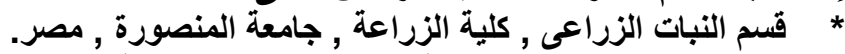

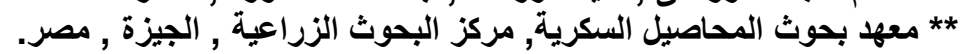

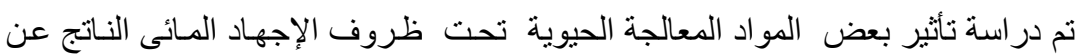

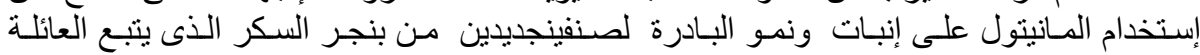

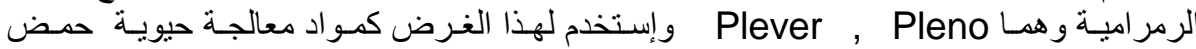
الأسكوربيك , حمض السليسليك , حمض الهيوميك ومستخلص الأعثاب البحرية .

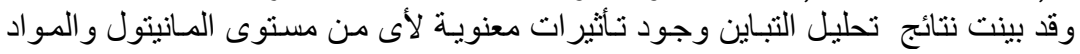

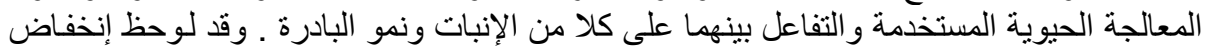

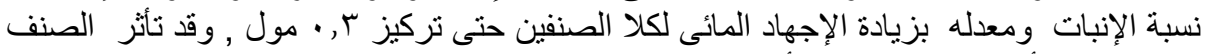

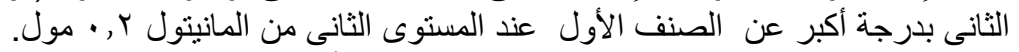

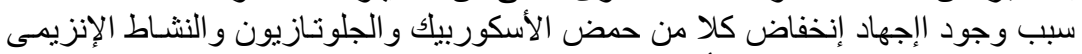

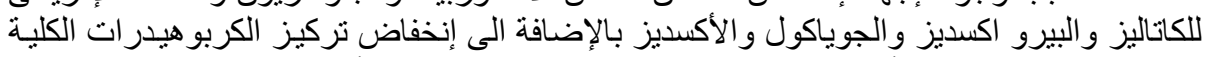

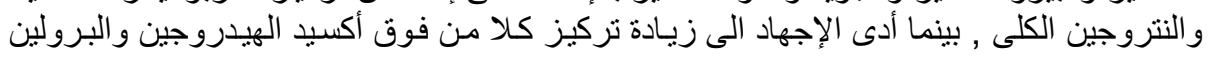

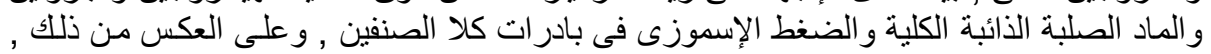

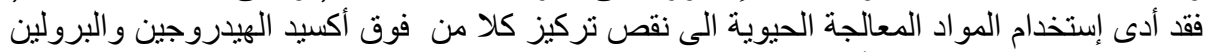

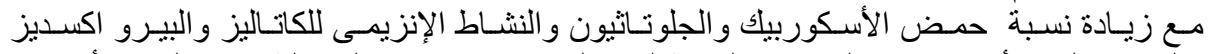

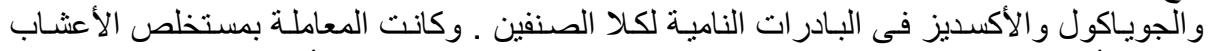

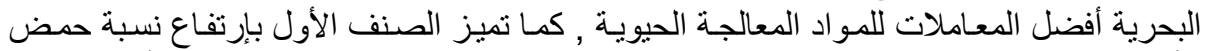
الأسكوربيك و الجلو تاثيون و النشاط الإنزيمى للكاتاليز و البيرو اكسديز والئيز والجوياكول والأكسديز عن

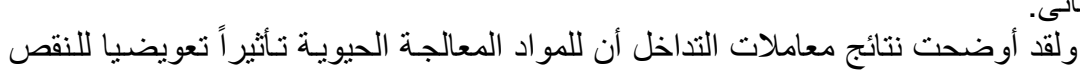
الصنف الأنانى.

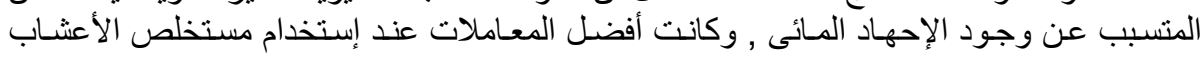

البحرية , وحمض الهيو ميك حتى تركيز r , • مول مانيتول.

قام بتحكيم البحث

كلية الزراعة - جامعة المنصورة

أ.د / رمضان عبد المنعم فودة

كلية الزراعة ـ جامعة عين شمس الزمس

أ.د / سعيد عواد شحاتة 